\title{
Uma solução genuína da equação de advecção difusão com forma sesquilinear para problema multi-fonte.
}

\author{
A genuine solution of the diffusion advection equation sesquilinear way to \\ multi-source problem.
}

\author{
Debora Lidia Gisch, Bardo Bodmann, Marco Túllio Menna Barreto de Vilhena
}

Programa de Pós-Graduação em Engenharia Mecânica, UFRGS, Porto Alegre, RS, Brasil debora.gisch@gmail.com, bejbodmann@gmail.com, mtmbvilhena@gmail.com

\begin{abstract}
Resumo
O presente trabalho é uma proposta para uma aproximação alternativa para o modelo de dispersão de poluentes, e incluir algumas características que podem ser associadas ao fenômeno de turbulência. Como ponto de partida nós consideramos dois axiomas que conduzem a um modelo e sua solução é compatível com as descrições de distribuições. No primeiro garantimos solução semi-positiva, como se espera de uma distribuição, considerando que o segundo axioma implementa estruturas compatíveis com estruturas coerentes através da implementação de formas sesquilinear.
\end{abstract}

Palavras-chave: Dispersão de Poluentes, Estruturas Coerentes, Formas Sesquilinear.

\begin{abstract}
The present work is a proposal for an alternative approach for pollution dispersion modelling, including some characteristics that may be associated to the phenomenon of turbulence. As a starting point we consider two axiomatic properties that shall lead to a model and its solution compatible with distributional descriptions. The first one states that a solution shall be semi-positive as expected for a distribution, whereas the second axiom demands for compatibility with coherent structures, which are implemented by the use of sesquilinear forms.
\end{abstract}

Keywords: Pollution Dispersion, Coherent Structures, Sesquilinear Forms. 


\section{Introdução}

A dispersão de poluentes é um fenômeno complexo dado que ao observar uma chaminé pode-se identificar diversas estruturas como vórtices e turbilhões, flutuações na densidade do poluente e na velocidade da dispersão Hussain e Fazle (1986). Um reflexo dessa complexidade é que, para reproduzi-la, há modelos que utilizam um grande número de parâmetros. Neste trabalho constróise um modelo de dispersão de poluentes que apresenta estas estruturas apenas introduzindo um coeficiente de difusão complexo, modificando, assim, o fechamento Fickiano Stull (1988).

\section{Metodologia}

O modelo analítico de dispersão de poluentes amplamente estudado é o da equação advecção-difusão Arya (1999) onde simplificações como as médias de Reynolds e o fechamento Fickiano são aplicados para que a solução seja facilmente obtida.

A equação é dada por,

$$
\frac{\partial C}{\partial t}+u \frac{\partial C}{\partial x}=K_{x} \frac{\partial^{2} C}{\partial x^{2}}+K_{y} \frac{\partial^{2} C}{\partial y^{2}}+K_{z} \frac{\partial^{2} C}{\partial z^{2}},
$$

onde $C\left[\mathrm{~g} / \mathrm{m}^{3}\right]$ concentração, $u[\mathrm{~m} / \mathrm{s}]$ velocidade na direção $x[m]$ e os $K^{\prime} s\left[m^{2} / \mathrm{s}\right]$ coeficientes de difusão das respectivas coordenadas espaciais. Sendo as condições, fluxo nulo no contorno, fontes contínuas com concentração nula no tempo inicial e constituída por três fontes pontuais alinhadas na coordenada y com distância de $0.1[\mathrm{~m}]$ entre elas. Descritas como:

$$
\begin{array}{r}
u C(0, y, z, t)=\dot{Q} \delta\left(y-y_{0}\right) \delta\left(z-H_{s}\right), \\
u C(0, y, z, t)=\dot{Q} \delta\left(y-y_{0}+0.1\right) \delta\left(z-H_{s}\right), \\
u C(0, y, z, t)=\dot{Q} \delta\left(y-y_{0}-0.1\right) \delta\left(z-H_{s}\right),
\end{array}
$$

onde $H_{S}$ é altura da fonte.

Para resgatar algumas características do fenômeno turbulento propomos a inclusão de uma fase na equação advecção-difusão tridimensional e transiente, já que (ECs) são características turbulentas dominadas pela fase. Assim resolve-se a equação advecção-difusão e ao obter-se a solução inclui-se uma modificação no fechamento Fickiano, através da substituição do coeficiente de difusão $K_{z}$ por um coeficiente de difusão complexo e formando a grandeza que representa a distribuição de poluentes de forma sesquilinear Bodmann et al. (2013). O complexo de uma constante é representado por $K_{z}=K_{z a}+i K_{z b}$, onde $K_{z a}$ é a parte real e $K_{z b}$ imaginária. Esse coeficiente complexo torna a solução da equação advecção-difusão complexa, ou seja a solução está no corpo dos complexos com módulo e fase. A distribuição da concentração deve ser real e

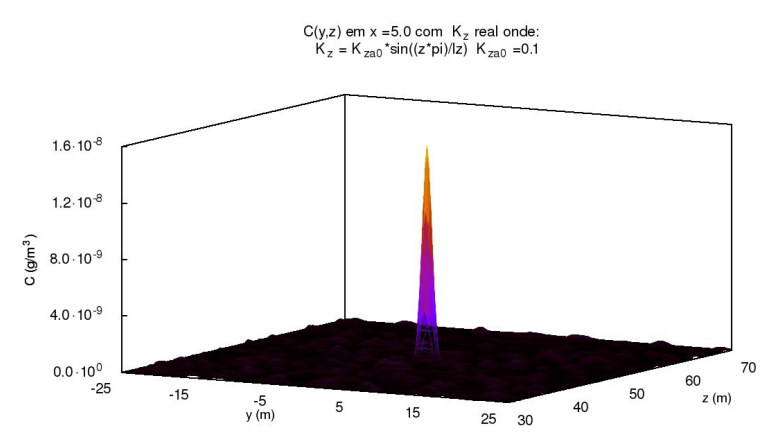

Figura 1: Distribuição $C(y, z) \operatorname{com} K_{z}$ real.

semi-positiva, portanto usa-se uma analogia aos fenômenos eletromagnéticos Jackson (1999), onde a densidade de energia é descrita de forma sesquilinear em termos do campo elétrico. Consequentemente a solução gera uma distribuição semi-positiva definida e não linear devolvendo assim uma característica qualitativa presente nos procedimentos de covariância de turbilhões. Então multiplicando a solução complexa $\mathscr{C}(x, y, z, t)$ pela sua solução complexa conjugada $\mathscr{C}^{*}(x, y, z, t)$

$$
\begin{gathered}
\mathscr{C}(x, y, z, t)=\sum_{n=1}^{\infty} \sum_{l=1}^{\infty} A_{n l} \mathscr{C}(x, t) \cos \left(\frac{n \pi}{L_{y}} y\right) \cos \left(\frac{l \pi}{L_{z}} z\right), \\
\mathscr{C}(x, t)=\int_{0}^{t} \frac{1}{2} e^{\frac{u}{2 K_{x}} e^{\left(\alpha-\frac{u^{2}}{4 K_{x}}\right) \tau} e^{-\left(\frac{x^{2}}{4 K_{x} \tau}\right)}} \\
{\left[\frac{x}{\sqrt{\pi K_{x} \tau^{3}}}-\frac{5 \sqrt{K_{x}} u}{\sqrt{\pi \tau}}\right] d \tau} \\
\alpha=-\left[\left(K_{z a}+i K_{z b}\right)\left(\frac{l \pi}{L_{z}}\right)^{2}+K_{y}\left(\frac{n \pi}{L_{y}}\right)^{2}\right]
\end{gathered}
$$

onde a diferença é que $K_{z}^{*}=K_{z a}-i K_{z b}$.

\section{Resultados e Discussões}

Foram determinadas uma série de distribuições espaciais e temporais-espaciais e comparadas com distribuições com o coeficiente de difusão real obtidas nas mesmas condições como em 1 e 2 . Mostramos a capacidade do modelo de reproduzir esses resultados sem nenhum comportamento turbulento apenas usando um coeficiente complexo com parte imaginária zero que nada mais é que a representação do conjunto dos reais no conjunto dos complexos.

Devido ao fato de por enquanto não existir uma prescrição de como determinar os parâmetros do modelo de forma à representar o fenômeno físico então 


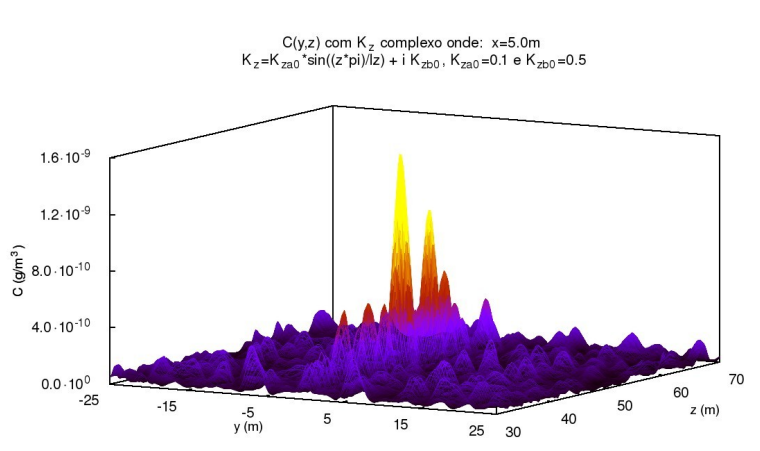

Figura 2: Distribuição $C(y, z)$ com $K_{z}$ complexo.
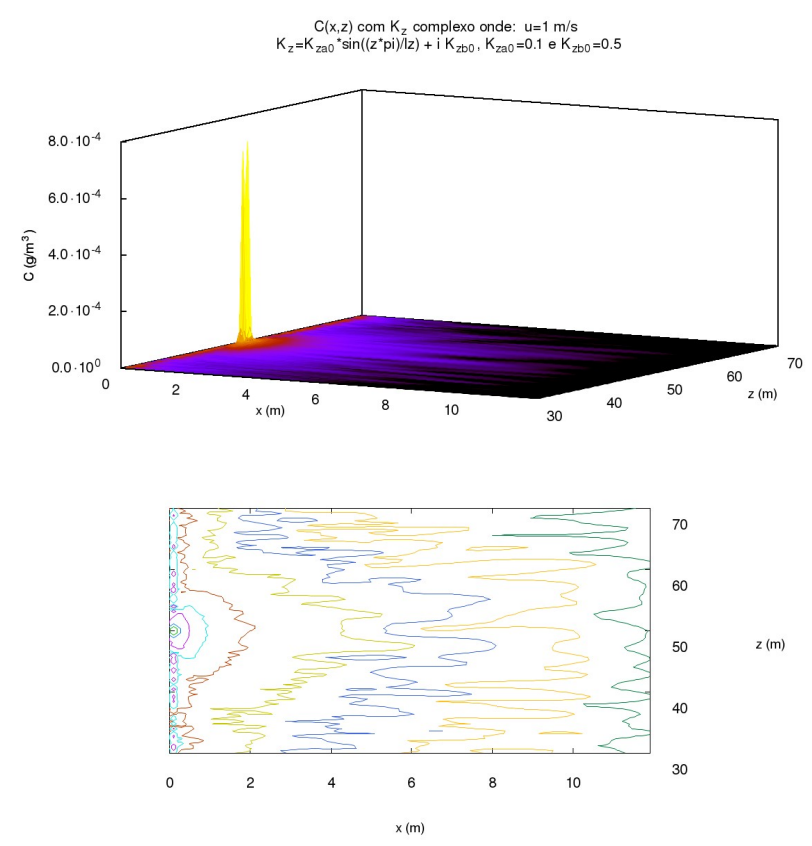

Figura 3: Distribuição e Mapa das concentrações $C(x, z)$ com vento fraco.

geram-se soluções para uma variedade de partes imaginárias dos coeficientes de difusão. Assim avalia-se o comportamento da solução ao variar a parte imaginária do coeficiente de difusão onde observa-se um alargamento da distribuição juntamente com oscilações o que concorda com o aumento do coeficiente, que no caso real quanto maior coeficiente de difusão maior é a dispersão.

O fenômeno de meandro observado na distribuição e no mapa 3, que em condições de vento fraco e próximo a fonte a dispersão de poluentes é irregular e indefinida onde a pluma está sujeita a ondulações horizontais à direção do vento também foi detectada como em Gisch et al. (2015). Este fenômeno não é observado na distribuição nem no mapa 4 em condições de vento forte.
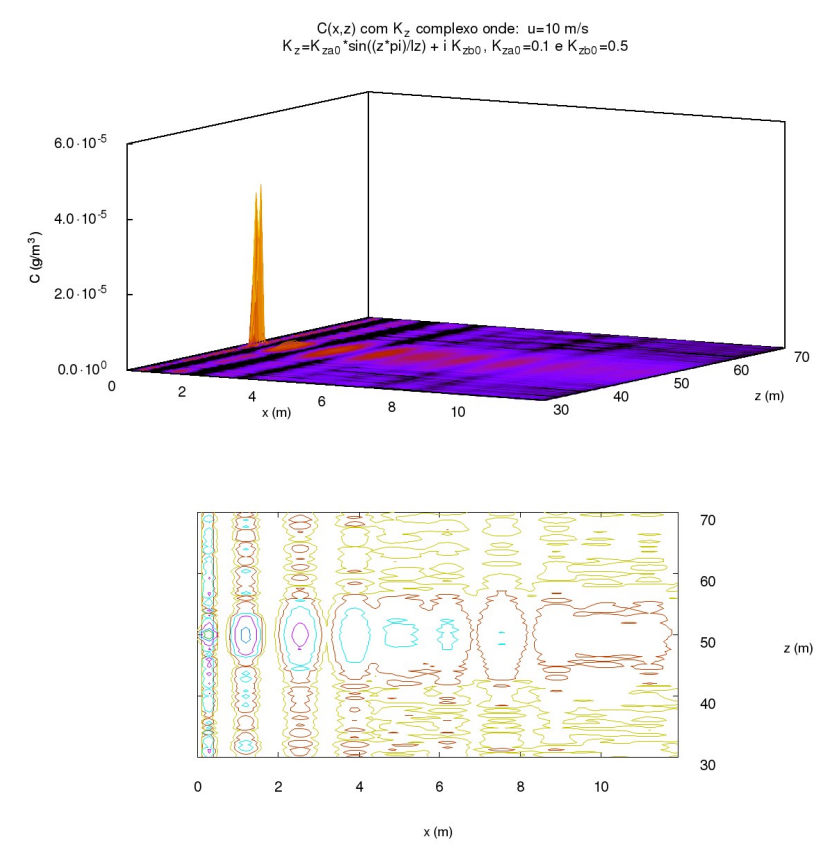

Figura 4: Distribuição e Mapa das concentrações $C(x, z)$ com vento forte.

\section{Conclusões}

O modelo de dispersão de poluentes com coeficiente de difusão real e complexo foi comparado para a mesma situação podendo observar qualitativamente a inclusão da fase no modelo. O modelo de dispersão de poluentes presente abre a possibilidade de descrever propriedades filigranas do fenômeno ao custo de poucos parâmetros. Descrições comparáveis com um modelo puramente real precisaria de um conjunto exorbitante de parâmetros para obter o mesmo efeito. Todos os resultados ainda são preliminares para garantir que esta será uma modificação justificada a partir de primeiros princípios, mas pretende-se estudar e incluir uma dependência temporal e espacial mais adequada no coeficiente de difusão e até mesmo substituir o coeficiente de difusão real por um complexo nas demais direções e subsequentemente validar os resultados com resultados experimentais.

\section{Agradecimentos}

Agradeço a CNPq a CAPES e a Linhares Geração S.A.

\section{Referências}

Arya, S. P. (1999). Air Pollution Meteorology and Dispersion. Oxford University Press, New York, USA.

Bodmann, B. E. J., Zabadal, J. R. S., Vilhena, M. T., Quadros, R. (2013). On coherent structures from a 
diffusion-like model. Em: Integral Methods in Science and Engineering, Springer New York Heidelberg Dordrecht London, pp. 1-10.

Gisch, D. L., Bodmann, B. E. J., Vilhena, M. T. (2015). Two reasons why pollution dispersion modelling needs sesquilinear forms. Em: Integral Methods in Science and Engineering, Springer International Publishing Switzerland, $\mathrm{p}$ Umpublished.

Hussain, A. K., Fazle, M. (1986). Coherent structures and turbulence. Journal of Fluid Mechanics, 173, 303, URL http://www.journals.cambridge. org/abstract_S0022112086001192.

Jackson, J. D. (1999). Classical electrodynamics, $3^{\circ}$ edn. Wiley, New York, NY, URL http://cdsweb. cern.ch/ record/490457.

Stull, R. B. (1988). An Introduction to Boundary Layer Meteorology. Kluwer Academic Publishers, Dordrecht, Holanda. 